\title{
Effect of Psycho-Social Rehabilitation on Psychological Well-being, Mental Health and Body Image Concern in Mastectomized Women
}

\author{
Toktam Sadat Jafar Tabatabaei ${ }^{1, *}$, Saloomeh Akbari ${ }^{2}$, Maryam Ghorbani ${ }^{3}$, Nahid Nasirfam ${ }^{4}$, \\ Fatemeh Esmaili ${ }^{5}$
}

${ }^{1}$ Assistant Professor, Department of Psychology, Birjand Branch, Islamic Azad University, Birjand, Iran

${ }^{2}$ MA of Consultation and Guidance, Department of Psychology, Roodehen Branch, Islamic Azad University, Roodehen, Iran

${ }^{3}$ Ph.D. Student of Educational Technology, Department of Educational Sciences, Faculty of Educational Science and Psychology, Allameh Tabatabaei University, Tehran, Iran

${ }^{4}$ Ph.D. Student of Educational Psychology, Department of Psychology, Birjand Branch, Islamic Azad University, Birjand, Iran

${ }^{5}$ Lecture, Department of Psychology, Bojnourd Branch, Islamic Azad University, Bojnourd, Iran

* Corresponding author: Toktam Sadat Jafar Tabatabaei, Assistant Professor, Department of Psychology, Birjand Branch, Islamic Azad University, Birjand, Iran. E-mail: toktamtabatabaee@yahoo.com

How to Cite this Article:

Jafar Tabatabaei TS, Akbari S, Ghorbani M, Nasirfam N, Esmaili F. Effect of Psycho-Social Rehabilitation on Psychological Wellbeing, Mental Health and Body Image Concern in Mastectomized Women. J North Khorasan Univ Med Sci. 2020;6(4):49-58.

DOI: $10.29252 /$ ijrn-06046

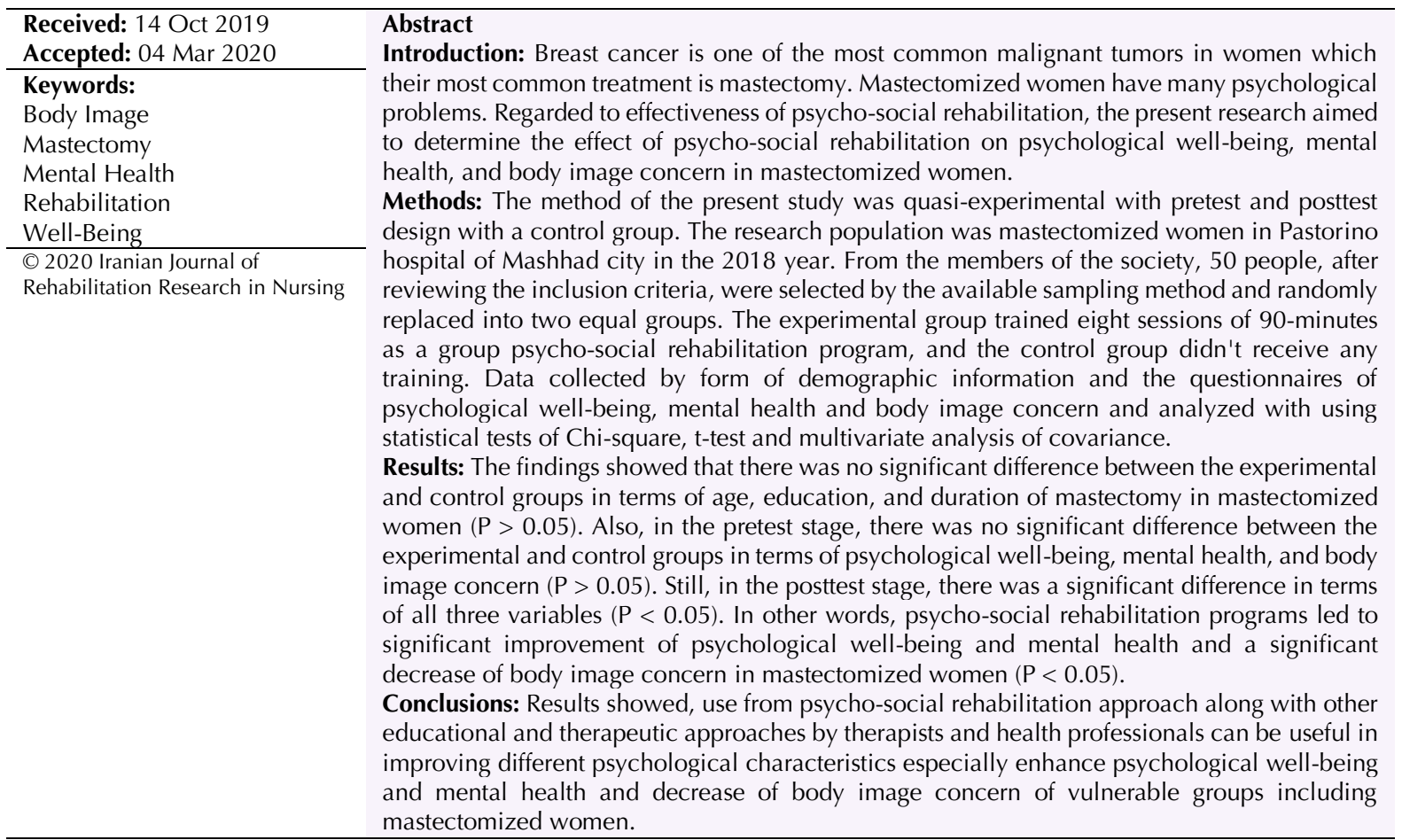

\section{Extended Abstract}

\section{OBJECTIVE}

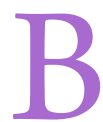

reast cancer is one of the most common malignant tumors in women, and this disease in all its forms and stages can have a profound effect on the family system [1]. One of the treatment methods for breast cancer is mastectomy [2], which affects the quality of the sexual relationship and disrupts sexual function. Mastectomized women often have a negative body image [4] and usually have psychological 
problems related to mental health and psychological well-being [5]. Body image concern is a multidimensional construct involving thoughts, feelings, beliefs, and behaviors related to the perceived body, and this construct refers to a negative assessment of body size, shape, and weight [7]. When a person experiences negative evaluation or being ridiculed by others, a negative body image is formed [6]. Also, Mental health is one of the most critical aspects of health, meaning the ability to balance and harmonious communication with others, to changing and modifies one's social environment, and to resolving personal conflicts and tendencies logically, equitably, and appropriately [8]. Today, mental health plays an important and prominent role in ensuring the dynamics and efficiency of the individual and society [9]. Also, psychological well-being is an attempt to realize one's talents and abilities, which include positive feelings and general satisfaction from life [11]. Mental well-being plays an active role in reducing stress and anxiety and improving the quality of life and health [12]. One of the therapeutic approaches to enhance many psychological problems is psycho-social rehabilitation [14]. This rehabilitation program emphasized the training of general individual and social skills and led to promotes mental health, enriches human relationships, and enhances health-related behaviors [16]. Psycho-social rehabilitation helps individuals to act positively and protect themselves and society from psycho-social trauma and improve the level of health [17]. According to the content, the present research aimed to determine the effect of psycho-social rehabilitation on psychological well-being, mental health, and body image concern in mastectomized women.

\section{METHODS}

The method of the present study was quasiexperimental with pretest and posttest design with experimental and control groups. The population was undergoing mastectomized women in Pastorino hospital of Mashhad city in the 2018 year. From the members of the community, 50 people after reviewing the inclusion criteria were selected by available sampling method and randomly were replaced into two equal groups including experimental and control groups (each group 25 people). The experimental group trained 8 sessions of 90-minutes (one sessions per week) as a group psycho-social rehabilitation program by a clinical psychologist with a degree of education period in a one of the psychological services clinics of Mashhad city and the control group was replaced on the waiting list for training. After reassuring subjects about ethical considerations and obtaining conscientious participation in research, for collect data used from the form of demographic information and the questionnaires of psychological well-being, mental health and body image concern. In the present study the reliability were calculated by Cronbach's alpha method for psychological well-being, mental health and body image concern in mastectomized women $0 / 88,0 / 93$ and $0 / 84$, respectively. Data after collected were analyzed with using statistical tests of Chi-square, t-test and multivariate analysis of covariance in SPSS-20 software in significant level of $\mathrm{P}<0 / 05$.

\section{RESULTS}

The results of the Chi-square test showed that between the experimental and control groups, there was no significant difference in terms of age, education, and duration of mastectomy in mastectomized women ( $\mathrm{P}>$ $0 / 05)$. The results of the $t$-test showed that between the experimental and control groups in the pretest stage, there was no significant difference in terms of psychological well-being, mental health, and body image concern in mastectomized women $(\mathrm{P}>0.05)$. Still, between the mentioned groups in the posttest stage, there was a significant difference in terms of all three variables of their psychological well-being, mental health, and body image concern $(\mathrm{P}<0.05)$ (Table 1$)$. In other words, psycho-social rehabilitation programs led to a significant improvement in psychological well-being and mental health and a significant decrease in body image concern in mastectomized women $(\mathrm{P}<0 / 05)$ (Table 2).

Table 1. T-Test Results

\begin{tabular}{|c|c|c|c|}
\hline Variables & Experimental Group & Control Group & P Value \\
\hline \multicolumn{4}{|c|}{ Psychological well-being } \\
\hline Pre-test & $37.29 \pm 6.35$ & $38.61 \pm 6.47$ & $>0.05$ \\
\hline Post-test & $46.17 \pm 6.82$ & $39.24 \pm 6.40$ & $<0.001$ \\
\hline \multicolumn{4}{|l|}{ Mental health } \\
\hline Pre-test & $43.26 \pm 7.29$ & $42.15 \pm 7.03$ & $>0.05$ \\
\hline Post-test & $34.77 \pm 5.24$ & $43.32 \pm 7.29$ & $<0.001$ \\
\hline \multicolumn{4}{|c|}{ Body image concern } \\
\hline Pre-test & $52.23 \pm 6.47$ & $50.96 \pm 6.11$ & $>0.05$ \\
\hline Post-test & $43.85 \pm 5.03$ & $51.22 \pm 3.38$ & $<0.001$ \\
\hline
\end{tabular}

Data are presents as Mean \pm SD.

Table 2. MANCOVA Results

\begin{tabular}{lcccccccc}
\hline Variables & Source & Sum of Squares & df & Mean Squares & F & P Value & Eta Squared & Test Power \\
\hline $\begin{array}{l}\text { Psychological well- } \\
\text { being }\end{array}$ & Group & 937.64 & 1 & 937.64 & 115.48 & $<0.001$ & 0.88 \\
Mental health & Group & 852.13 & 1 & 852.13 & 109.37 & $<0.001$ & 0.86 \\
Body image concern & Group & 682.15 & 1 & 682.15 & 99.70 & $<0.001$ & 0.81 & 0.93 \\
\hline
\end{tabular}




\section{CONCLUSIONS}

The results of the present study showed that training of psycho-social rehabilitation led to improve psychological well-being and mental health and decrease body image concern in mastectomized women. Considering to the physical and psychological problems of mastectomized women and the effectiveness of psycho-social rehabilitation programs to improve personal and social living conditions, it is recommended that to enhance different psychological characteristics including improving psychological well-being and mental health and decrease of body image concern of vulnerable groups including mastectomized women to use from psycho-social rehabilitation approach along with other educational and therapeutic strategies.

\section{Ethical Considerations}

This research has a code of ethics number of IR.IAUBirjand.1397.43 from Islamic Azad University of Birjand branch. Also, it performs Helsinki ethics protocols, the information of subjects remains anonymous, and after the study, all this information will be protected. Also, all participants signed the research consent form and described for all of them the purpose, importance, and necessity of research.

\section{Funding or Supports}

This study had no sponsors and was conducted with a personal cost.

\section{Author's Contribution}

In the present article, Salome Akbari was responsible for data collection and entering data to a computer, Maryam Ghorbani and Nahid Nasirfam was accountable for collecting theoretical and research backgrounds and assistance in writing the article, Fatemeh Esmaili was responsible for assist in the implementation of the intervention and Toktam Sadat Jafar Tabatabaei was accountable for the implementation of response and writing, submitting and editing the article.

\section{Conflict of Interest}

Based on the authors declare in this study, there is no conflict of interest.

\section{Applicable Remarks}

In this study, mastectomized women had problems in the field of psychological well-being, mental health, and body image, and training psycho-social rehabilitation program could improve their psychological well-being and mental health and reduce their body image concern. Then, learning psycho-social rehabilitation by nurses, therapists, and health experts and its proper use can play an essential role in improving psychological characteristics related to health. As a result, the mentioned rehabilitation program can play a critical role in policies of preventive, health, and treatment-related to vulnerable groups, including mastectomized women in improving psychological well-being and mental health and reduce their body image concern.

\section{Acknowledgments}

The authors of this article are gratitude and appreciation from the authorities of Pastorno hospital of Mashhad city due to collaborating with researchers and from participants due to active participation. 


\section{تاثير توانبخشى روانى - اجتماعى بر بهزيستى روانشناختى، سلامت روان و نكرانى از تصوير بدنى در زنان ماستكتومىشده}

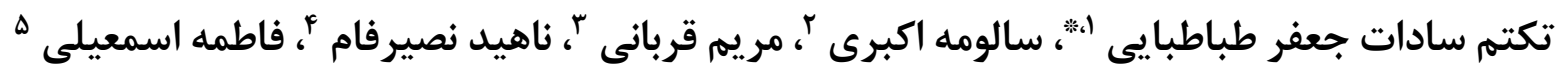

' استاديار، كروه روانشناسى، واحد بيرجند، دانشكاه آزاد اسلامى، بيرجند، ايران

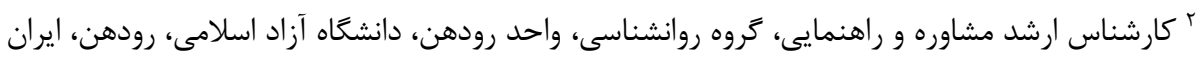

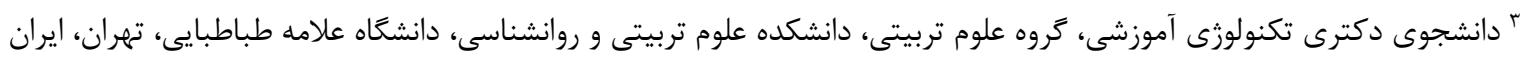

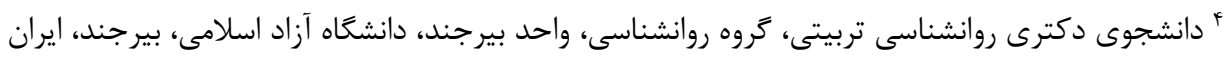

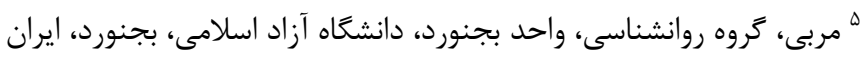

* نويسنده مسئول: تكتم سادات جعفر طباطبايى، استاديار، گروه روانشناسى، واحد بيرجند، دانشعاه آزاد اسلامى، بيرجند، ايران. ايميل: toktamtabatabaee@yahoo.com

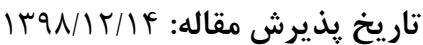

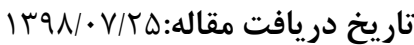

جكيده مقدمه: سرطان يستان از شايع ترين تورمورهاى بدخيم در زنان است كه روش درمان رايج آن ماستكتومى مىباشد. زنان ماستكتومىشده داراى مشكلات روانشناختى

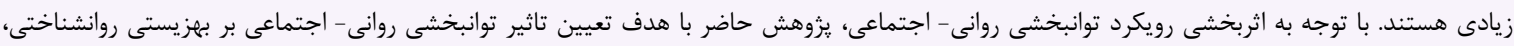
سلامت روان و نَخرانى از تصوير بدنى در زنان ماستكتومىشده انجام شد. روش كار: روش مطالعه حاضر نيمهتجربى با طرح پِيشآزمون و پِّآزمون با گروه كنترل بود. جامعه يروهش زنان ماستكتومىشده در بيمارستان ياستورنو شهر

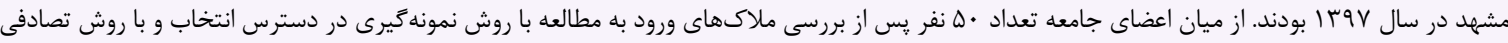

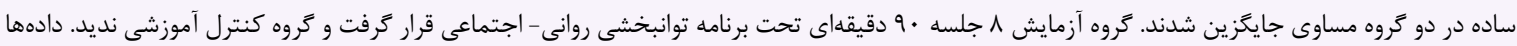

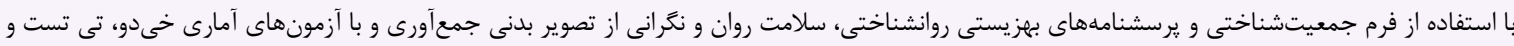
تحليل كوواريانس קندمتغيرى تحليل شدند. يافتهها: نتايج نشان داد كه بين كروههاى آزمايش و كنترل از نظر سن، تحصيلات و مدت زمان كذشته از عمل ماستكتومى در زنان ماستكتومى آشده تفاوت معنى

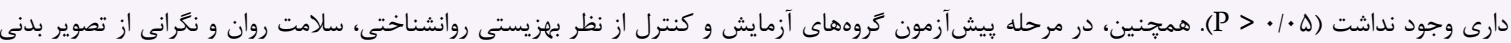

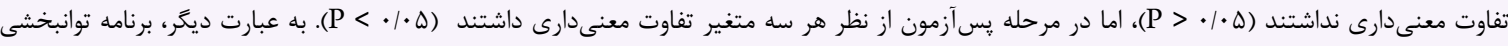

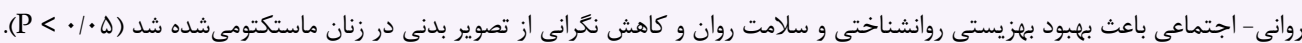

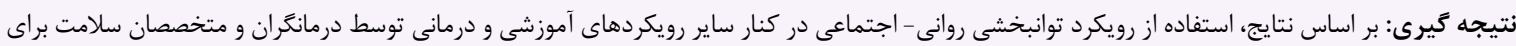

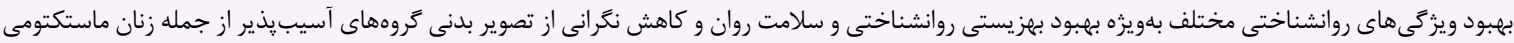
شده مىتواند موثر واقع شود. كليدوازمها: بهزيستى، تصوير بدنى، توانبخشى، سلامت روان، ماستكتومى مودى تمامى حقوق نشر براى انجمن علمى يرستارى ايران محفوظ است.

سيستم خانوادگى بر جاى بحذارد [1]. سرطان پِّتان دومين علت

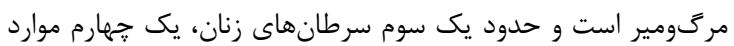

سرطان يستان از شايعترين تورمورهاى بدخيم در زنان است و اين بيمارى در تمامى انواع و مراحل خود مىتواند تاثيرات شديدى بر بر بر برى 


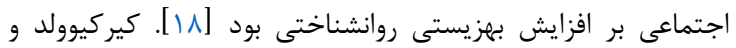

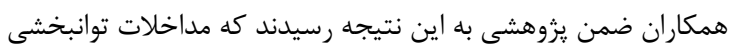

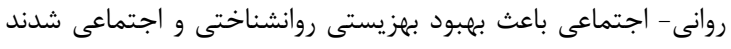

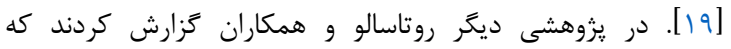

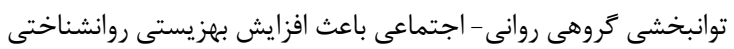

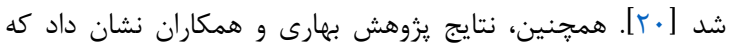

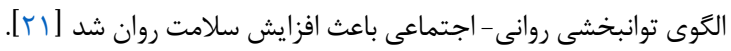

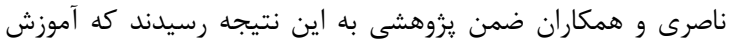

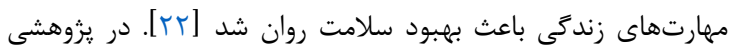

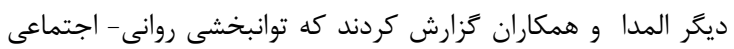

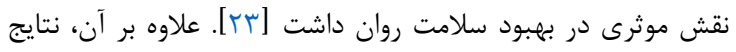

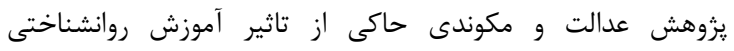

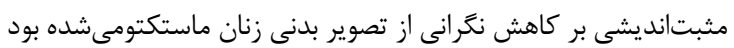

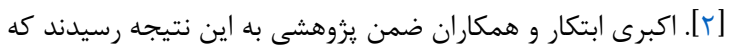

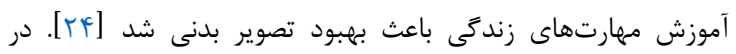

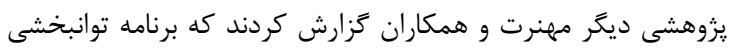

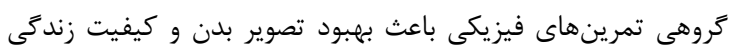

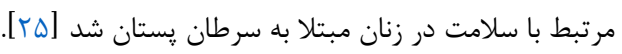

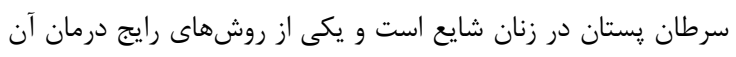

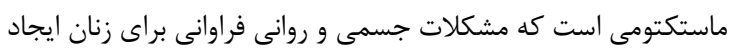

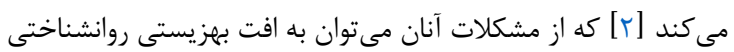

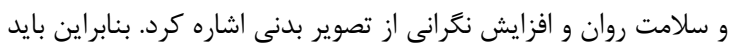

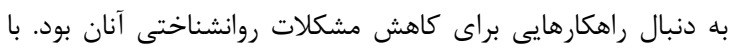

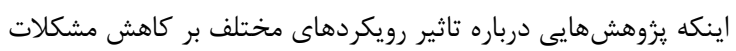

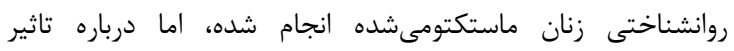

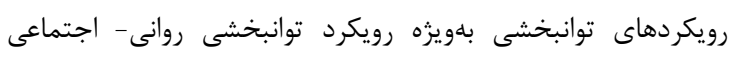

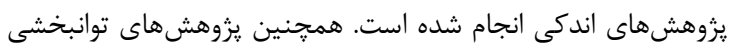

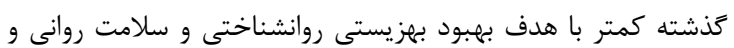

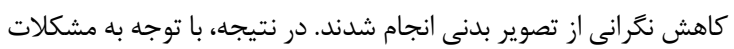

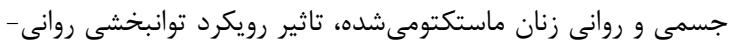

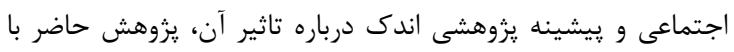

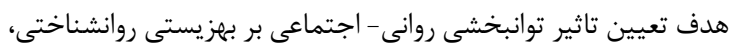

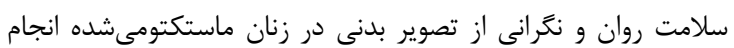

\section{روش كار}

اين مطالعه نيمهتجربى با طرح ييش آزمون و پِيسآزمون با كروه كنترل

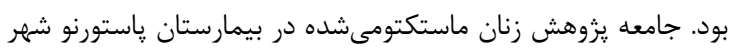

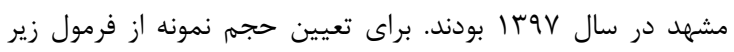

$$
\mathrm{n}=\frac{2 \sigma^{2}\left(z_{1-\frac{\alpha}{2}+} z_{1-\beta}\right)^{2}}{d^{2}}
$$

استفاده شد.

با توجه به فرمول بالا و طبق نتايج يزوهش خلعتبرى و همكاران [وب]

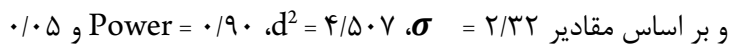
a=

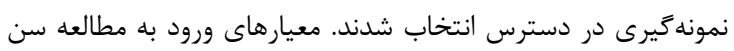

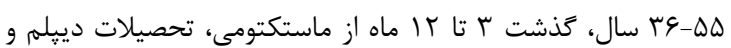

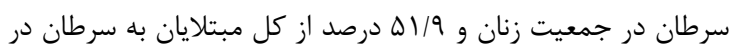

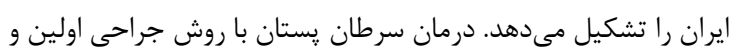

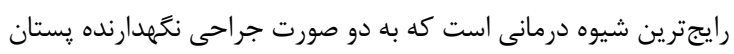

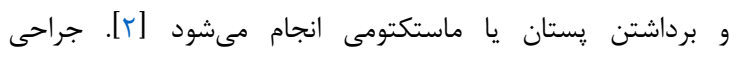

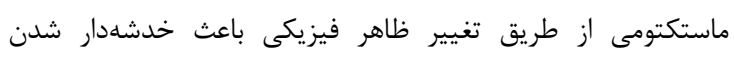

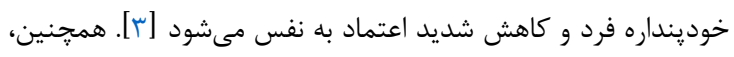

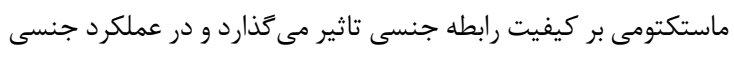

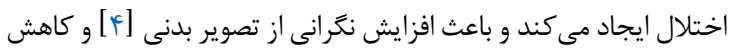

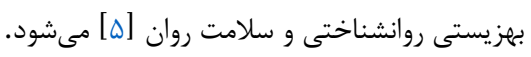

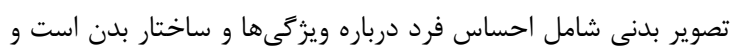

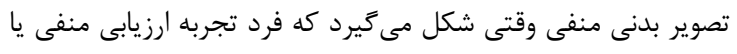

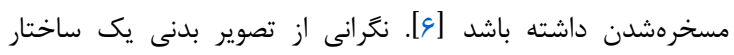

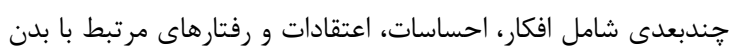

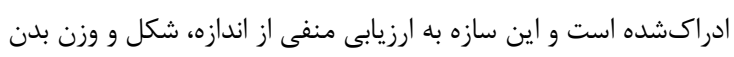

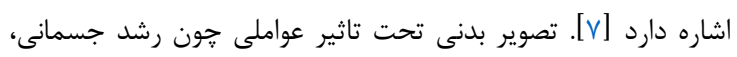

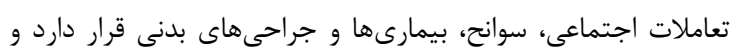

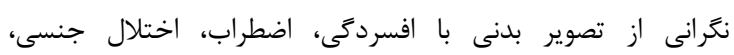

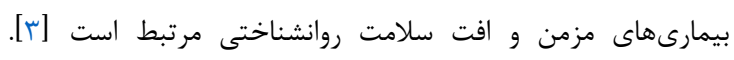

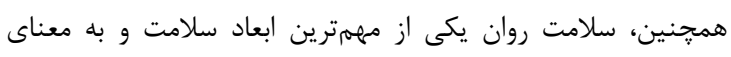

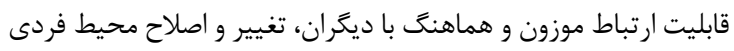

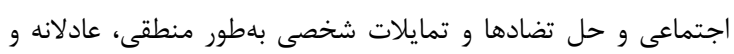

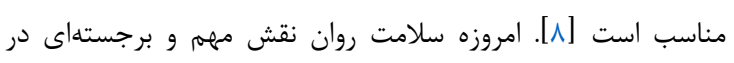

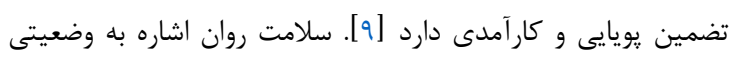

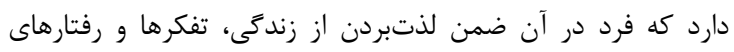

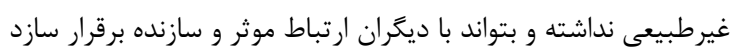

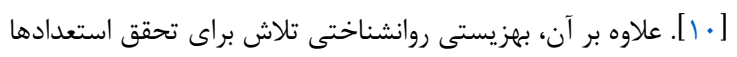

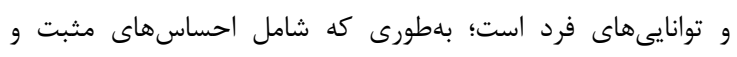

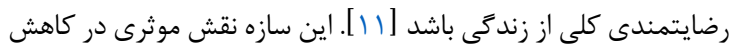

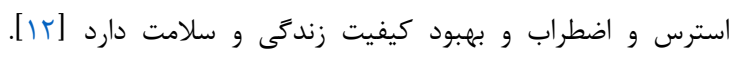

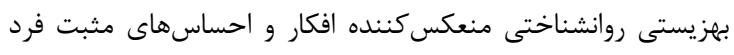

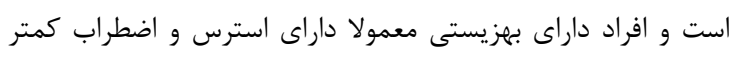

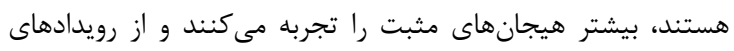

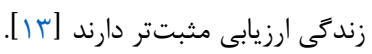

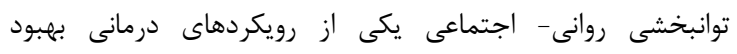

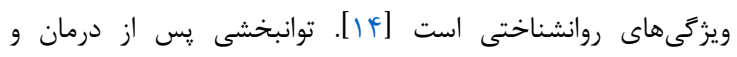

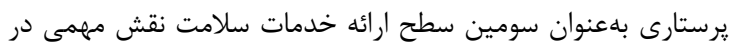

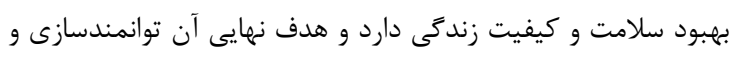

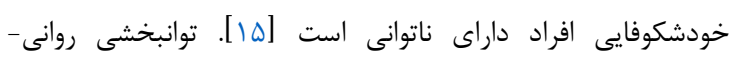

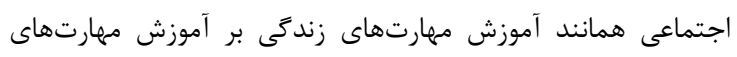

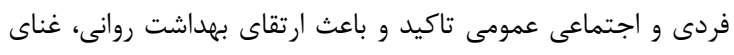

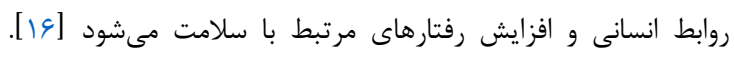

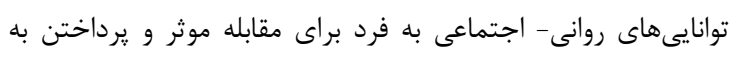

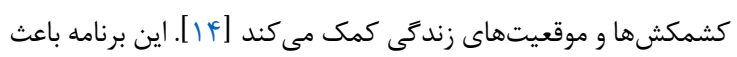

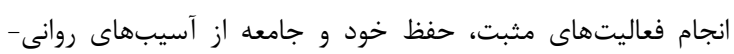

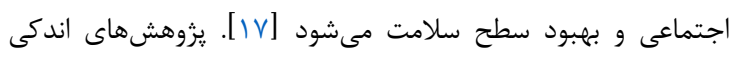

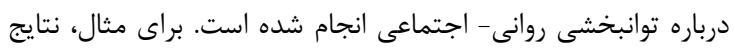

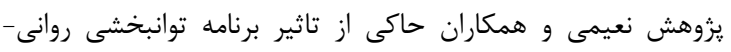


كروه آزمايش و گروه ديخر بهعنوان گروه كنترل انتخاب شد. به

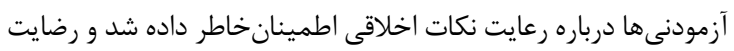

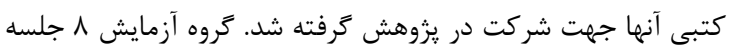

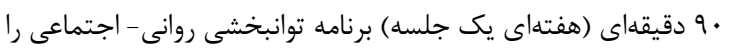

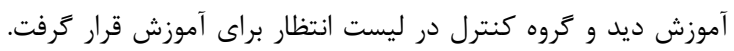

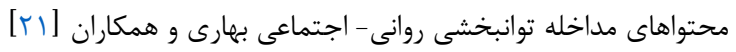
در جدول اكزارش شده است.
بالاتر از آن، عدم اعتياد و مصرف داروهاى روانيزشكى و عدم استفاده

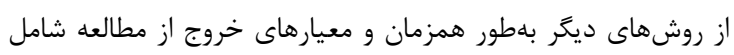

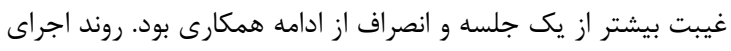

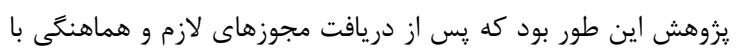

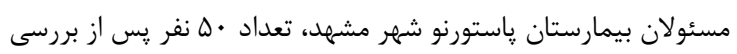

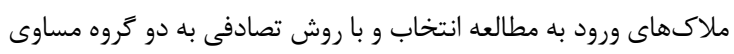

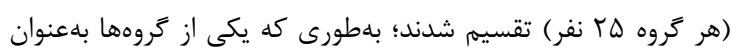

\begin{tabular}{|c|c|}
\hline محتوى & جلسات \\
\hline \multirow{2}{*}{\multicolumn{2}{|c|}{ و تصوير بدنى إد مباط مستحكم و حمايتكر بين افراد، ايجاد برداشت مثبت از عضويت در كروه، آكاهى از اهداف جلسات آموزشى و آشنايى با بهزيستى روانشناختى، سلامت روان }} \\
\hline & \\
\hline ايجاد جو مثبت و شكستن سردى بين اعضا و شناخت راهكارهاى بهبود سلامت و كيفيت زندگى & دوم \\
\hline آشنايى با علل و عوامل موثر در اختلال هاى روانشناختى و آَاهى از علائم و نشانههاى بهزيستى روانشناختى، سلامت روان و نكرانى از تصوير بدنى & سوم \\
\hline شناخت مسئله، يادكيرى مهارتهاى حل مسئله نظاممند و آموزش راهكارهاى بهبود وضعيت روانشناختى & جهارم \\
\hline توسعه مهارتهاى حل مسئله نظاممند و مهارتهاى رهبرى و مديريت و آموزش استفاده از راه حلهاى سازكارانه به جاى راه حلهاى عادتى براى مقابله با مشكلات & ينجم \\
\hline شناخت مهارتها و توانايىهاى فردى واقعى، آشنايى با ايدهآل ها و آرزوهاى خود و آكاهى از وظايف خود از ديدكاه خود و ديكران & ششم \\
\hline شناخت نقش جنسيتى زنانه در جامعه، آكاهى از روابط اجتماعى و اهميت آن در زندگى، داشتن باورهاى مثبت در زندكى و بررسى و بهبود احساسها و هيجانها & 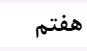 \\
\hline مرور و جمعبندى جلسات قبل توسط آزمودنىها با كمك درمانكر & 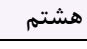 \\
\hline
\end{tabular}

نَخرانى بيشتر از تصوير بدنى مىباشد. روايى صورى و محتوايى ابزار

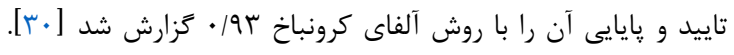

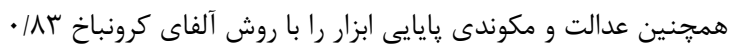

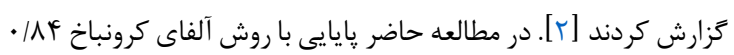
بدست آمد.

\section{يافتهها}

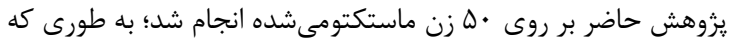

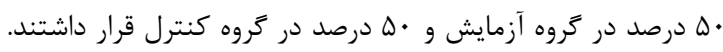

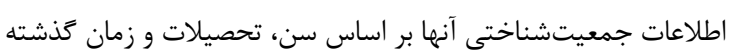
از عمل ماستكتومى در جدول r كزارش شده است.

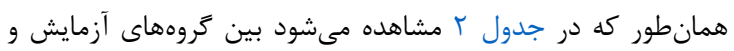

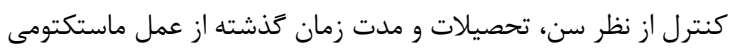

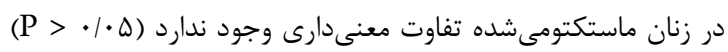

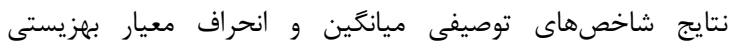

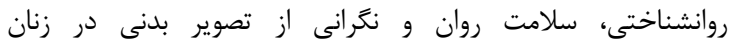

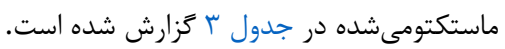

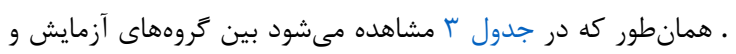

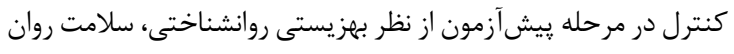

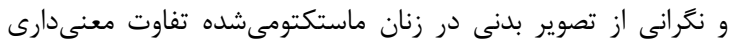

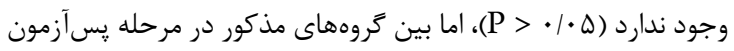

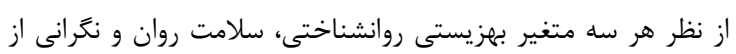

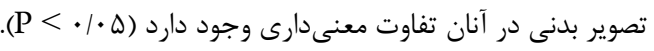

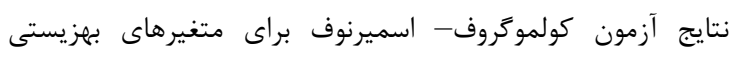

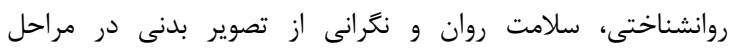

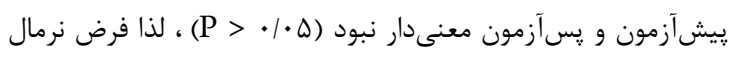

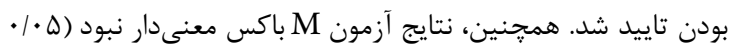
(P >

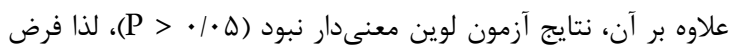

برنامه توانبخشى روانى - اجتماعى توسط يك متخصص روانشناسى داسى داني

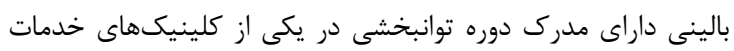

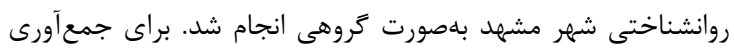

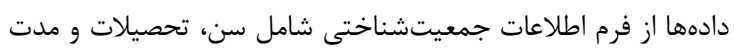

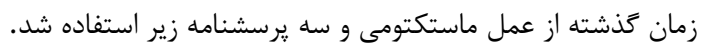

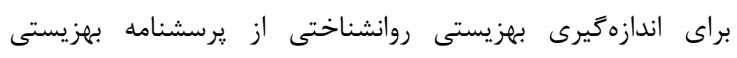

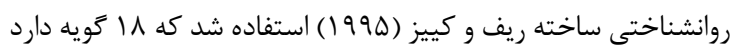

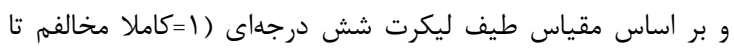

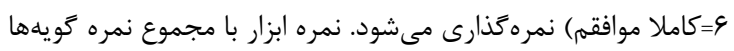

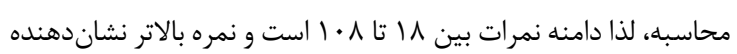

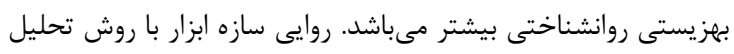

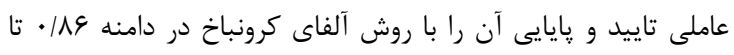

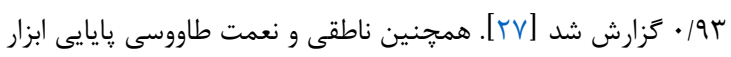

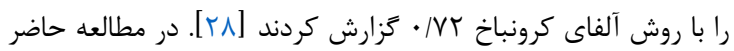

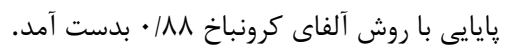

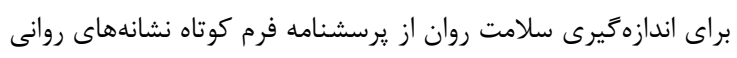

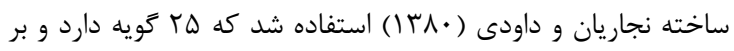

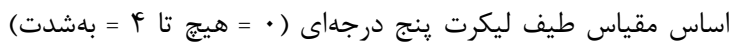

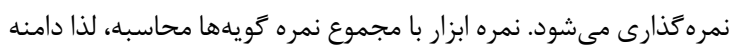

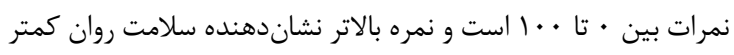

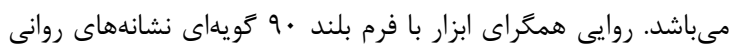

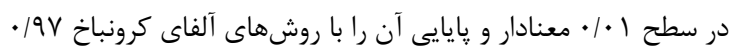

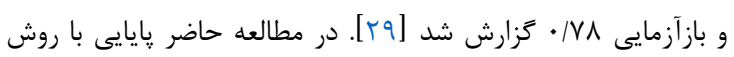

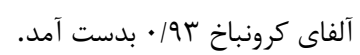

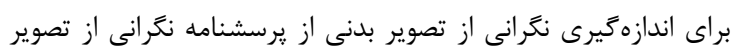

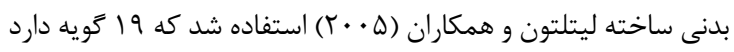

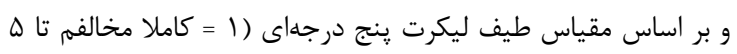

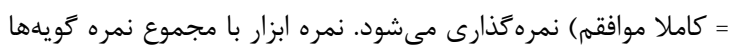

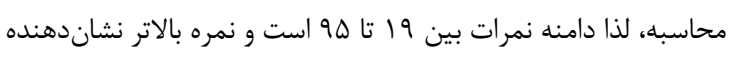


روانشناختى، سلامت روان و نكرانى از تصوير بدنى در زنان

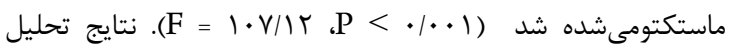

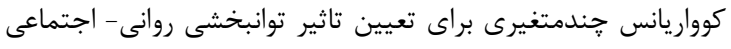

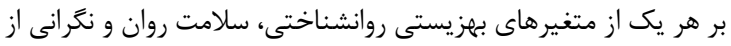

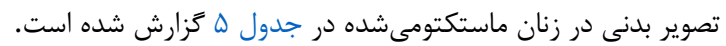

برابرى واريانسها تاييد شد. بنابراين مفروضههاى تحليل كوواريانس

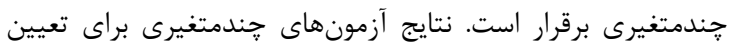

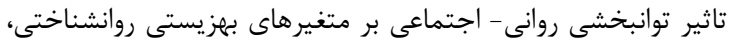

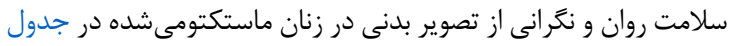
عز عزارش شده است.

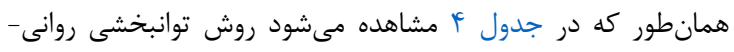

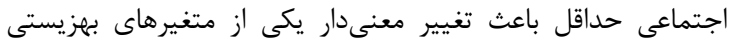

جدول r. فراوانى و درصد فراوانى ويثزى هاى جمعيتشناختى واحدهاى يروهش

\begin{tabular}{|c|c|c|c|}
\hline معنىدارىChi-square & كروه كنترل & كروه آزمايش & متغيرها \\
\hline \multirow[t]{5}{*}{.$/ 901$} & & & سن (سال) \\
\hline & $(T \cdot) \Delta$ & $(19)^{f}$ & re-f. \\
\hline & $(19) F$ & $(r \cdot) \Delta$ & $f \backslash-F \Delta$ \\
\hline & (T) 1 & (14) 9 & $+\varphi-Q$ \\
\hline & (Tr) $\wedge$ & $(r \wedge) V$ & $\Delta 1-\Delta \Delta$ \\
\hline \multirow[t]{5}{*}{.$/ 9 r F$} & & & تحصيلات \\
\hline & $(T M) \wedge$ & $(T Y)^{G}$ & دييلم \\
\hline & $(Y F)^{Q}$ & $(r \wedge) V$ & كاردانى \\
\hline & $(Y \Lambda) V$ & $(r T) \wedge$ & كارشناسى \\
\hline & $(19) f^{f}$ & $(19) F$ & كارشناسى ارشد \\
\hline \multirow[t]{4}{*}{$\cdot / \wedge \Delta \cdot$} & & & زمان كذشته از عمل (ماه) \\
\hline & $(F \wedge) \| r$ & $(f \cdot))$. & $k-9$ \\
\hline & $(Y \Lambda) \vee$ & $(\Pi r) \wedge$ & $V-9$ \\
\hline & $(T F)^{9}$ & $(r \wedge) V$ & $1 \cdot-15$ \\
\hline
\end{tabular}

جدول ب. نتايج شاخصهاى توصيفى بهزيستى روانشناختى، سلامت روان و نكرانى از تصوير بدنى در زنان ماستكتومىشده

\begin{tabular}{|c|c|c|c|}
\hline معنى دارى T-Test & كروه كنترل & كروه آزمايش & متغير ها \\
\hline & & & بهزيستى روانشناختى \\
\hline $\mathrm{P}>\cdot / \cdot \Delta$ & $r N|q| \pm \varepsilon / 4 V$ & rV/\&rq/r山 & ي پيشآزمون \\
\hline \multirow[t]{2}{*}{$\mathrm{P}<\cdot / \cdot 1$} & $r q / r F \pm g / 4$. & $r \& / / V \pm \& / A T$ & 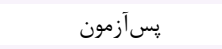 \\
\hline & & & سلامت روان \\
\hline $\mathrm{P}>\cdot / \cdot \Delta$ & $F r / l \Delta \pm V / \cdot r$ & $F T / T \& \pm V / T q$ & ي پيشآزمون \\
\hline \multirow[t]{2}{*}{$\mathrm{P}<\cdot / \cdot 1$} & $R r / r T \pm V / T q$ & $r F / V V \pm \Delta / T F$ & يس آزمون \\
\hline & & & نكرانى از تصوير بدنى \\
\hline $\mathrm{P}>\cdot / \cdot \Delta$ & $0 \cdot 199 \pm 9 / 11$ & $\Delta T / T r \pm g / F V$ & بيش آزمون \\
\hline $\mathrm{P}<\cdot / \cdot \cdot 1$ & $\Delta 1 / r r \pm T / r \Lambda$ & $r r / \Lambda \Delta \pm \Delta / \cdot r$ & يسآزمون \\
\hline
\end{tabular}

جدول F. نتايج آزمونهاى هندمتغيرى براى تعيين تاثير توانبخشى روانى - اجتماعى بر متغيرها در زنان ماستكتومىشده

\begin{tabular}{|c|c|c|c|c|c|}
\hline توان آزمون & مجذور اتا & PValue & ضريبF & آماره & آزمونها \\
\hline .194 & $\cdot / 1 \Delta \Delta$ & $.1 \cdot+1$ & $1 \cdot V / I T$ & $\cdot / \Lambda 9$ & اثر پِيلايى \\
\hline .194 & $\cdot / \Lambda \Delta$ & $\cdot / \cdot 1$ & $1 \cdot V / 1 T$ & $\cdot|r|$ & لامبداى ويلكز \\
\hline .194 & $\cdot / \Lambda \Delta$ & $\cdot \mid \cdot 1$ & $1 \cdot V / 1 T$ & I/AF & اثر هاتلينت \\
\hline.$/ 94$ & $\cdot / \wedge \Delta$ & $\cdot / \cdot 1$ & $1 \cdot V / I T$ & l/AF & بزرىترين ريشه روى \\
\hline
\end{tabular}

جدول ه. نتايج تحليل كوواريانس خندمتغيرى براى تعيين تاثير توانبخشى روانى - اجتماعى بر هر يك از متغيرها در زنان ماستكتومىشده

\begin{tabular}{|c|c|c|c|c|c|c|c|c|}
\hline توان آزمون & مجذور اتا & P Value & ضريب F & ميانگين مجذورات & درجه آزادى & مجموع مجذورات & منبع اثر & متغير ها \\
\hline .199 & $\cdot / \Lambda \Lambda$ &.$/ \cdot 1$ & $\| \Delta / 4 \lambda$ & qTV/G4 & 1 & qTV/G4 & كروه & بهزيستى روانشناختى \\
\hline . & $\cdot 1 \Lambda 9$ & $\cdot 1 \cdot \cdot 1$ & $1.9 / \% V$ & $\Lambda \Delta T / I T$ & 1 & $\Lambda \Delta T / I T$ & كروه & سلامت روان \\
\hline$\cdot 119$ & $\cdot|1|$ & $\cdot 1 \cdot \cdot 1$ & १९/V. & GNT/ID & 1 & GAT/IQ & تروه & نكرانى از تصوير بدنى \\
\hline
\end{tabular}

سلامت روان و نكَانى از تصوير بدنى در زنان ماستكتومىشده شده

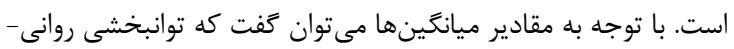

همانطور كه در جدول ه مشاهده مىشود روش توانبخشى روانى - مهى اجتماعى باعث تغيير معنى دار هر سه متغير بهزيستى روانشناختى، 
اينكه آموزش توانبخشى روانى- اجتماعى احتمالا مىتواند منشأ

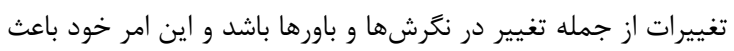

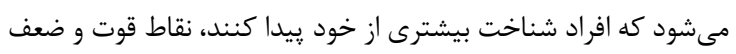

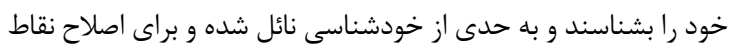

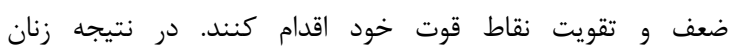

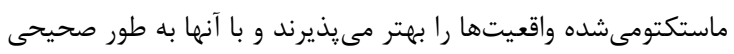

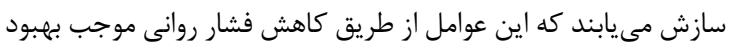
سلامتى روانى مىشوند.

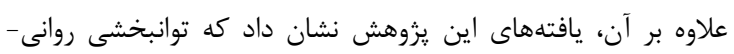

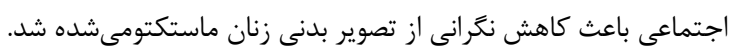

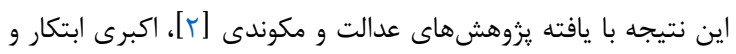

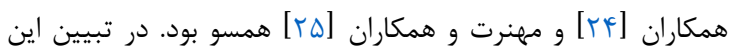

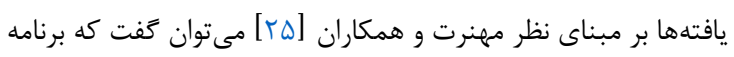

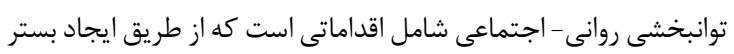

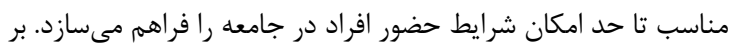

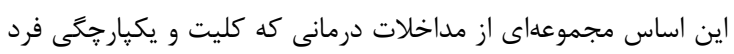

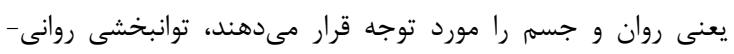

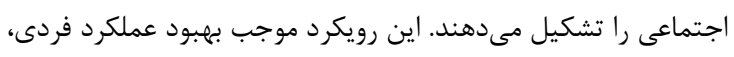

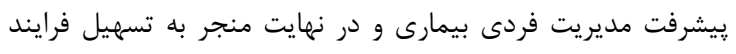

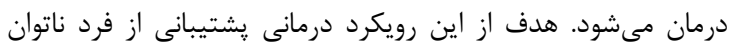

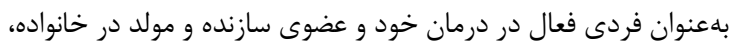

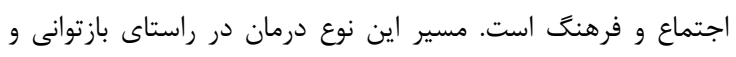

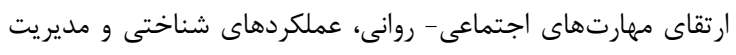

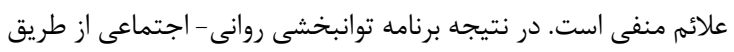

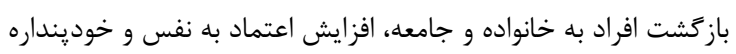

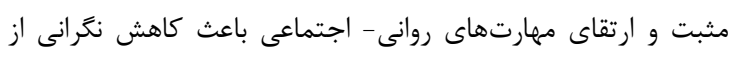

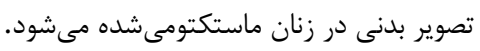

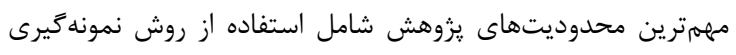

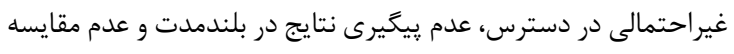

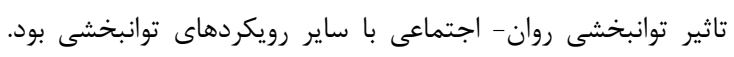

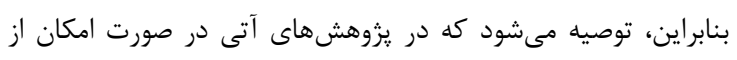

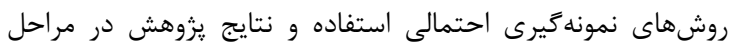

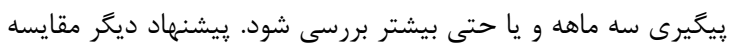

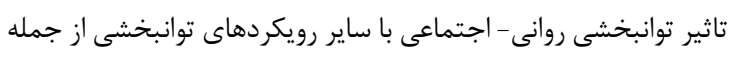

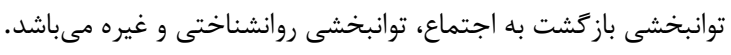

\section{نتيجه تيرى}

بلهور كلى يافتهاى اين يزووهش نشان دادند كه توانبخشى روانى -

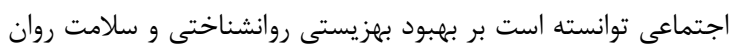

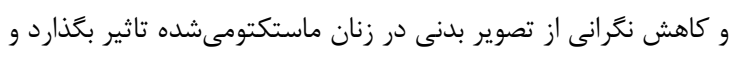

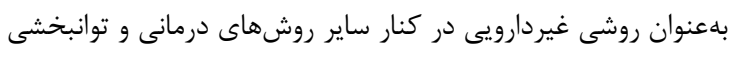

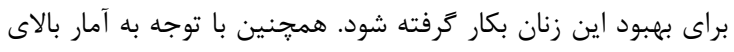

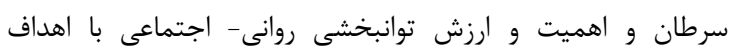

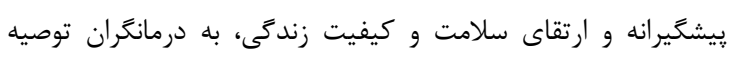

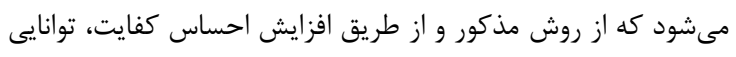

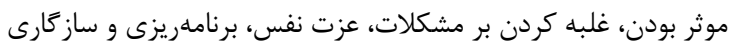

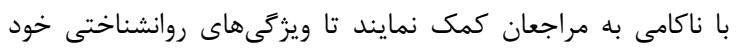

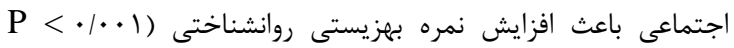

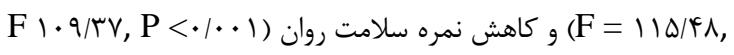

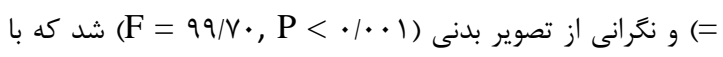

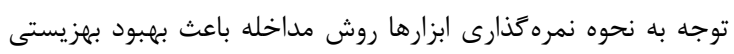

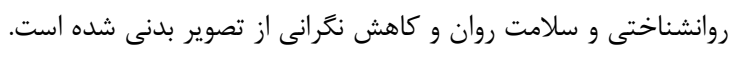

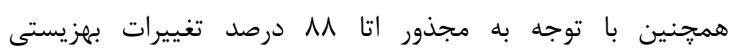

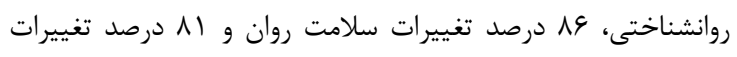

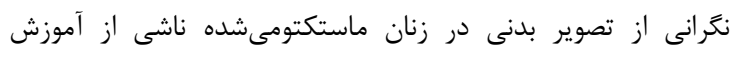
توانبخشى روانى - اجتماعى است.

با توجه به آمار نسبتا بالاى سرطان يستان در زنان و استفاده از روش

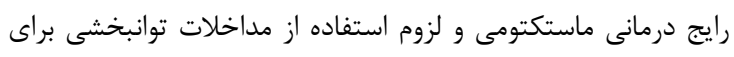

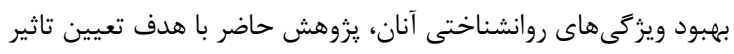

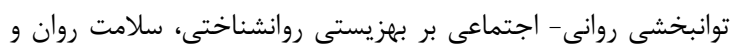

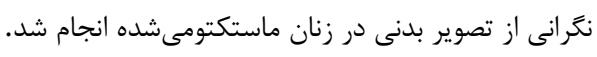

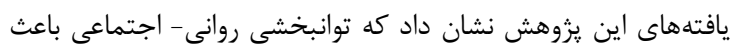

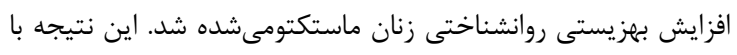

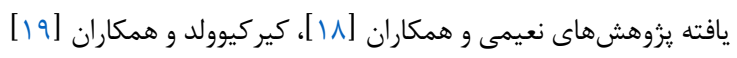

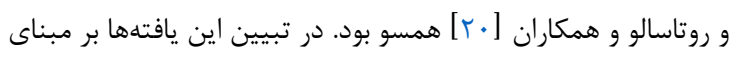

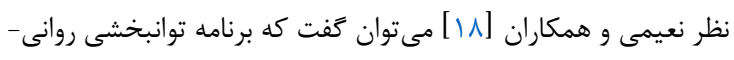

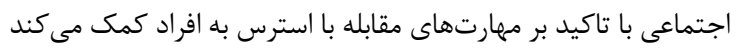

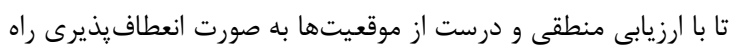

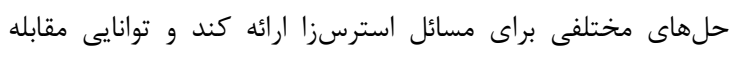

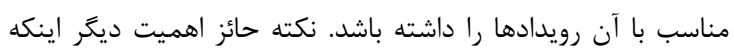

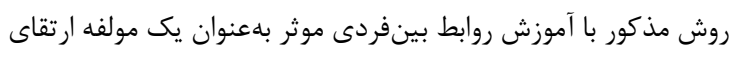

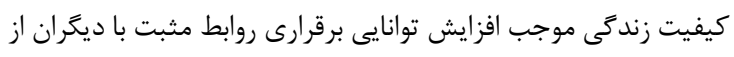

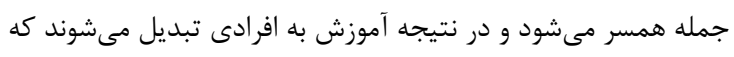

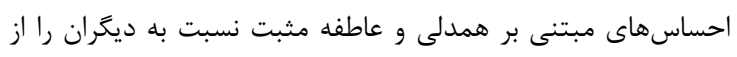

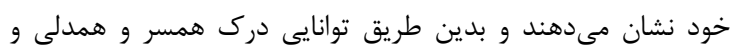

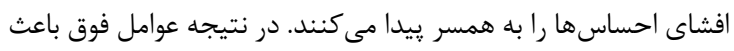

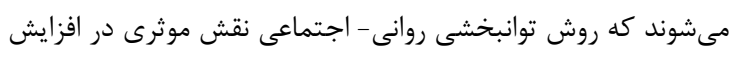

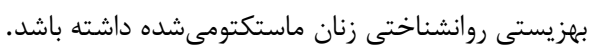

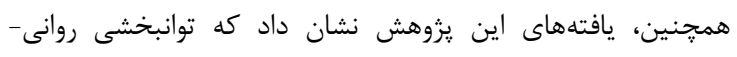

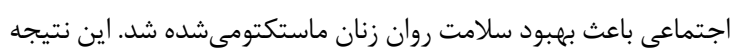

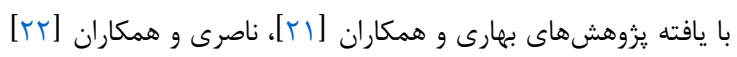

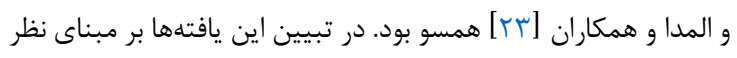

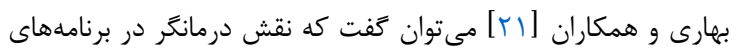

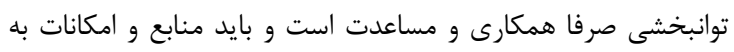

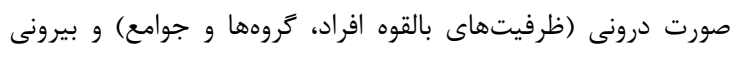

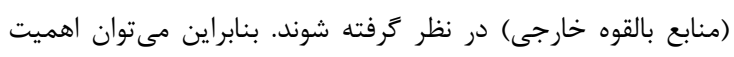

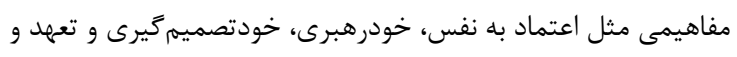

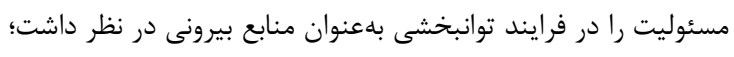

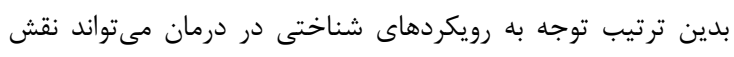

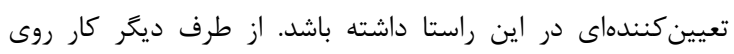

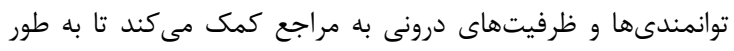

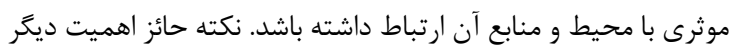


كمك در اجراى مداخله و تكتم سادات جعفر طباطبايى مسئول اجراى

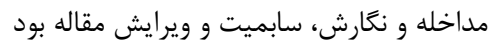

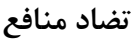

بر اساس اظهارنظر نويسندگًان در اين مطالعه هيجَّونه تضاد منافعى نصاف

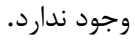

كاربرد عملى مطالعه

در اين مطالعه زنان ماستكومى داراى مشكلاتى در زمينه بهزيس مانستى

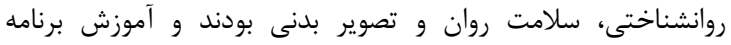

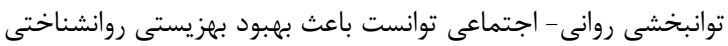

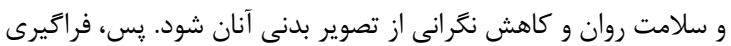

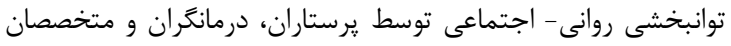

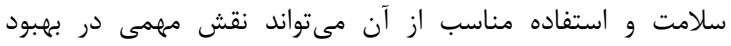

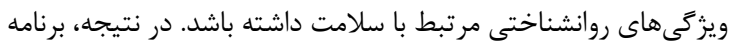

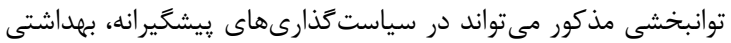

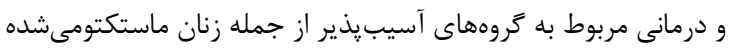

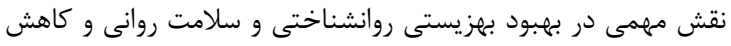
نغرانى از تصوير بدنى داشته باشد.

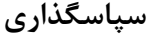

نويسند أن مقاله از مسئولان بيمارستان پاستورنو شهر مشهد به دليل

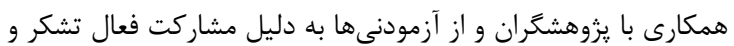

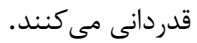

\section{References}

1. Yu TJ, Liu YY, Hu X, Di GH. Survival following breastconserving therapy is equal to that following mastectomy in young women with early-stage invasive lobular carcinoma. Eur J Surg Oncol. 2018;44(11):1703-7. doi: 10.1016/j.ejso.2018.06.026 pmid: 30029824

2. Edalat A, Makvandi B. Effectiveness of positive thinking training on stress, rumination and body image concern in mastectomized women. (Persian). IJRN. 2019;6(1):6976. doi: $0.21859 / \mathrm{ijrn}-06108$

3. Almahariq MF, Quinn TJ, Siddiqui Z, Jawad MS, Chen PY, Gustafson GS, et al. Breast conserving therapy is associated with improved overall survival compared to mastectomy in early-stage, lymph node-negative breast cancer. Radiother Oncol. 2020;142:186-94. doi: 10.1016/j.radonc.2019.09.018 pmid: 31615634

4. Menon AS, O'Mahony M. Women's body image following mastectomy: Snap shots of their daily lives. Appl Nurs Res. 2019;47:4-9. doi: 10.1016/j.apnr.2019.03.002 pmid: 31113545

5. Shahbazi S, Woods SJ. Influence of physician, patient, and health care system characteristics on the use of outpatient mastectomy. Am J Surg. 2016;211(4):802-9. doi: 10.1016/j.amjsurg.2015.10.021 pmid: 26792275

6. Fogelkvist M, Gustafsson SA, Kjellin L, Parling T. Acceptance and commitment therapy to reduce eating disorder symptoms and body image problems in patients with residual eating disorder symptoms: A randomized

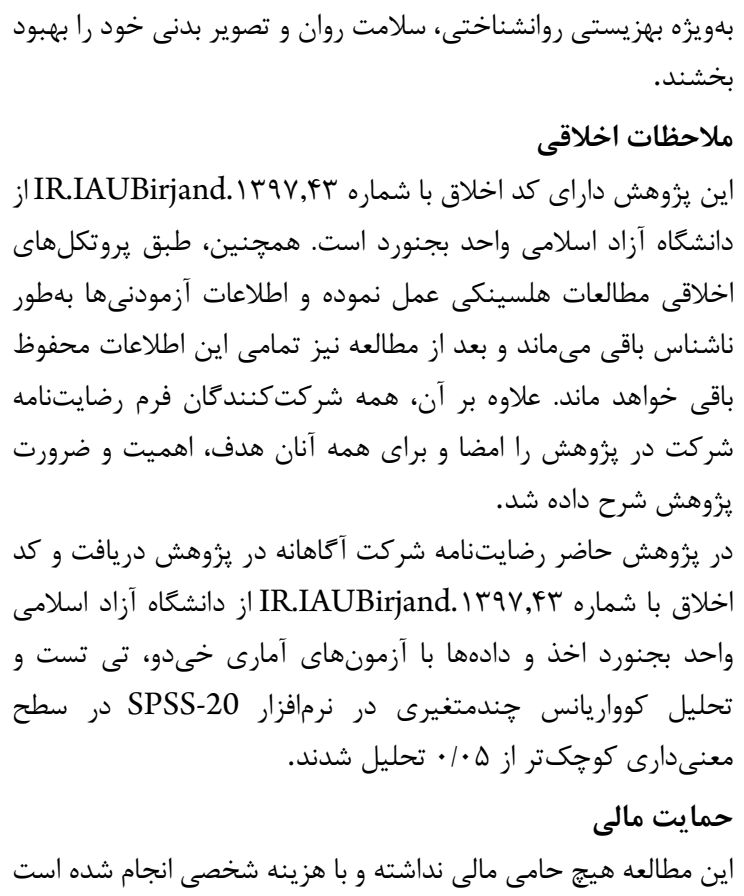

در مقاله حاضر سالومه اكبرى مسئول كردآورى دادهها و ورود دادهها

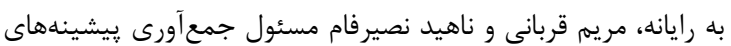
نظرى و يزوهشى و كمك در نغارش مقاله، فاطمه اسمعيلى مسئول

controlled trial. Body Image. 2020;32:155-66. doi: 10.1016/j.bodyim.2020.01.002 pmid: 32000093

7. Cerea S, Bottesi G, Grisham JR, Ghisi M. Non-weightrelated body image concerns and Body Dysmorphic Disorder prevalence in patients with Anorexia Nervosa. Psychiatry Res. 2018;267:120-5. doi: 10.1016/j.psychres.2018.05.068 pmid: 29886274

8. Iasiello M, van Agteren J, Keyes CLM, Cochrane EM. Positive mental health as a predictor of recovery from mental illness. J Affect Disord. 2019;251:227-30. doi: 10.1016/j.jad.2019.03.065 pmid: 30927584

9. Zahir Izuan A, Shamsul Azhar S, Tan MKS, SyedSharizman SAR. Neighbourhood influences and its association with the mental health of adolescents in Kuala Lumpur, Malaysia. Asian J Psychiatr. 2018;38:35-41. doi: 10.1016/j.ajp.2018.10.018 pmid: 30408711

10. Adjorlolo S, Aziato L, Akorli VV. Promoting maternal mental health in Ghana: An examination of the involvement and professional development needs of nurses and midwives. Nurse Educ Pract. 2019;39:105-10. doi: 10.1016/j.nepr.2019.08.008 pmid: 31446219

11. Twenge JM, Martin GN. Gender differences in associations between digital media use and psychological well-being: Evidence from three large datasets. J Adolesc. 2020;79:91-102.

doi: 10.1016/j.adolescence.2019.12.018 pmid: 31926450

12. Lin Z, Chen F. Evolving parent-adult child relations: location of multiple children and psychological well-being 
of older adults in China. Public Health. 2018;158:117-23. doi: $10.1016 /$ j.puhe.2018.02.024 pmid: 29588068

13. Lee $\mathrm{CH}$, Sibley CG. Sleep duration and psychological well-being among New Zealanders. Sleep Health. 2019;5(6):606-14. doi: 10.1016/j.sleh.2019.06.008 pmid: 31377250

14. Rispaud SG, Rose J, Kurtz MM. The relationship between change in cognition and change in functional ability in schizophrenia during cognitive and psychosocial rehabilitation. Psychiatry Res. 2016;244:145-50. doi: 10.1016/j.psychres.2016.07.033 pmid: 27479105

15. Yeterian JD, Berke DS, Litz BT. Psychosocial rehabilitation after war trauma with adaptive disclosure: Design and rationale of a comparative efficacy trial. Contemp Clin Trials. 2017;61:10-5. doi: 10.1016/j.cct.2017.07.012 pmid: 28713032

16. Prat G, Escandell MJ, Garcia-Franco M, Martin-Martinez JR, Tortades I, Vilamala S, et al. Gender differences in social functioning in people with schizophrenia in psychosocial rehabilitation services using onedimensional and multidimensional instruments. Compr Psychiatry. 2018;87:120-2. doi: 10.1016/j.comppsych.2018.10.004 pmid: 30340017

17. Antai-Otong D. Psychosocial Recovery and Rehabilitation. Nurs Clin North Am. 2016;51(2):287-97. doi: 10.1016/j.cnur.2016.01.011 pmid: 27229282

18. Naeimi Gh, Zahrakar K, Mohsenzadeh F, Pirsaghi F. The effectiveness of psycho-social empowerment program in reducing parental stress and enhancing psychological well-being of parents with mentally retarded children. (Persian). Empower Except Child. 2017;8(22):96-108.

19. Kirkevold M, Kildal Bragstad L, Bronken BA, Kvigne K, Martinsen R, Gabrielsen Hjelle E, et al. Promoting psychosocial well-being following stroke: study protocol for a randomized, controlled trial. BMC Psychol. 2018;6(1):12. doi: 10.1186/s40359-018-0223-6 pmid: 29615136

20. Routasalo PE, Tilvis RS, Kautiainen H, Pitkala KH. Effects of psychosocial group rehabilitation on social functioning, loneliness and well-being of lonely, older people: randomized controlled trial. J Adv Nurs. 2009;65(2):297305. doi: 10.1111/j.1365-2648.2008.04837.x pmid: 19054177

21. Bahari E, Raheb Gh, Eghlima M. The dffect of the psychological-social empowering model on the mental health of women with uusbands with chronic mental disease. (Persian). J Soc Work Res. 2019;4(13):37-71. doi: 10.22054/rjsw.2017.10222

22. Naseri L, Azizpour Y, Saiedy M, Rahmani M, Syaehmiri K. The effect of life skills education on the mental health of First year student in Ilam university of medical science in 2013 year. (Persian). JMED. 2015;10(3):237-45.

23. Almada AL, Casquinha P, Cotovio V, Heitor Dos Santos MJ, Caixeiro A. The potential role of psychosocial rehabilitation in palliative care. J R Coll Physicians Edinb. 2018;48(4):311-7. doi: 10.4997/JRCPE.2018.405 pmid: 30488884

24. Akbari Abtekar M, Hassanzadeh R, Barimani P. Effectiveness of life skills training on body image and depressive champ two high school students of Babol. (Persian). J Psychol New Ideas. 2017;1(2):19-26.

25. Mehnert A, Veers S, Howaldt D, Braumann KM, Koch U, Schulz KH. Effects of a physical exercise rehabilitation group program on anxiety, depression, body image, and health-related quality of life among breast cancer patients. Onkologie. 2011;34(5):248-53. doi: 10.1159/000327813 pmid: 21577030

26. Khalatbari J, Hemmati Sabet V, Mohammadi H. Effect of compassion-focused therapy on body image and marital satisfaction in women with breast cancer. (Persian). Iran Quarter J Breast Disease. 2018;11(3):8-20. doi: 10.30699/acadpub.ijbd..11.3.7

27. Ryff CD, Keyes LM. The structure of psychological wellbeing revisited. J Pers Soc Psychol. 1995;69(4):719-27. doi: 10.1037/0022-3514.69.4.719

28. Nateghi M, Nemattavousi $M$. The mediating role of selfesteem and self-regulation in relation of basic psychological needs and psychological wellbeing. (Persian). Psychol Stud. 2019;15(2):145-63. doi: 10.22051/psy.2019.24941.1853

29. Najarian B, Davodi I. Construction and validation SCL-25 (shortened form(SCL-90-R)). (Persian). J Psychol. 2001;5(2):136-49.

30. Littleton HL, Axsom D, Pury CL. Development of the body image concern inventory. Behav Res Ther. 2005;43(2):229-41. doi: 10.1016/j.brat.2003.12.006 pmid: 15629752 